\title{
Calibrating the surface brightness - color relation for late-type red giants stars in the visible domain using VEGA/CHARA interferometric observations
}

\author{
N. Nardetto ${ }^{1}$, A. Salsi ${ }^{1}$, D. Mourard ${ }^{1}$, V. Hocdé ${ }^{1}$, K. Perraut ${ }^{2}$, A. Gallenne ${ }^{1,3,4,5}$, A. Mérand ${ }^{6}$, D. Graczyk ${ }^{3,4}$, \\ G. Pietrzynski ${ }^{4}$, W. Gieren ${ }^{3}$, P. Kervella ${ }^{7}$, R. Ligi ${ }^{8}$, A. Meilland ${ }^{1}$, F. Morand ${ }^{1}$, P. Stee ${ }^{1}$, \\ I. Tallon-Bosc ${ }^{9}$, and T. ten Brummelaar ${ }^{10,11}$ \\ ${ }^{1}$ Université Côte d'Azur, Observatoire de la Côte d'Azur, CNRS, Laboratoire Lagrange, France \\ e-mail: Nicolas. Nardetto@oca.eu \\ ${ }^{2}$ Univ. Grenoble Alpes, CNRS, IPAG, 38000 Grenoble, France \\ ${ }^{3}$ Departamento de Astronomía, Universidad de Concepción, Casilla 160-C, Concepción, Chile \\ ${ }^{4}$ Nicolaus Copernicus Astronomical Center, Polish Academy of Sciences, ul. Bartycka 18, 00-716 Warszawa, Poland \\ ${ }^{5}$ Unidad Mixta Internacional Franco-Chilena de Astronomía (CNRS UMI 3386), Departamento de Astronomía, Universidad de \\ Chile, Camino El Observatorio 1515, Las Condes, Santiago, Chile \\ ${ }^{6}$ European Southern Observatory, Alonso de Córdova 3107, Casilla 19001, Santiago 19, Chile \\ ${ }^{7}$ LESIA (UMR 8109), Observatoire de Paris, PSL, CNRS, UPMC, Univ. Paris-Diderot, 5 place Jules Janssen, 92195 Meudon, \\ France \\ ${ }^{8}$ Osservatorio Astronomico di Brera, Via E. Bianchi 46, 23807 Merate, Italy \\ ${ }^{9}$ Université de Lyon, Université Lyon 1, Ecole Normale Supérieure de Lyon, CNRS, Centre de Recherche Astrophysique de Lyon \\ UMR5574, 69230, Saint-Genis-Laval, France \\ ${ }^{10}$ Georgia State University, PO Box 3969, Atlanta GA 30302-3969, USA \\ ${ }^{11}$ CHARA Array, Mount Wilson Observatory, 91023 Mount Wilson CA, USA
}

Received 7 February 2020 / Accepted 14 May 2020

\begin{abstract}
Context. The surface brightness - color relationship (SBCR) is a poweful tool for determining the angular diameter of stars from photometry. It was for instance used to derive the distance of eclipsing binaries in the Large Magellanic Cloud (LMC), which led to its distance determination with an accuracy of $1 \%$.

Aims. We calibrate the SBCR for red giant stars in the $2.1 \leq V-K \leq 2.5$ color range using homogeneous VEGA/CHARA interferometric data secured in the visible domain, and compare it to the relation based on infrared interferometric observations, which were used to derive the distance to the LMC.

Methods. Observations of eight G-K giants were obtained with the VEGA/CHARA instrument. The derived limb-darkened angular diameters were combined with a homogeneous set of infrared magnitudes in order to constrain the SBCR.

Results. The average precision we obtain on the limb-darkened angular diameters of the eight stars in our sample is $2.4 \%$. For the four stars in common observed by both VEGA/CHARA and PIONIER/VLTI, we find a $1 \sigma$ agreement for the angular diameters. The SBCR we obtain in the visible has a dispersion of 0.04 magnitude and is consistent with the one derived in the infrared ( 0.018 magnitude). Conclusions. The consistency of the infrared and visible angular diameters and SBCR reinforces the result of $1 \%$ precision and accuracy recently achieved on the distance of the LMC using the eclipsing-binary technique. It also indicates that it is possible to combine interferometric observations at different wavelengths when the SBCR is calibrated.
\end{abstract}

Key words. techniques: interferometry - stars: atmospheres - stars: distances - binaries: eclipsing - stars: late-type stars: fundamental parameters

\section{Introduction}

In the era of precision cosmology, it is essential to determine the Hubble constant to an accuracy of $2 \%$ or better (Komatsu et al. 2011; Freedman \& Madore 2010). Recently, Riess et al. (2019) achieved a $1.9 \%$ precision by relying on the distance to the Large Magellanic Cloud (LMC), which is the best anchor point for the cosmic distance scale (Schaefer 2008; Walker et al. 2012; Riess et al. 2016). Late-type eclipsing-binary systems provide the opportunity of measuring accurate distances by combining the linear diameter (derived from the light curve and velocimetry) and angular diameters (derived from a surface-brightness color relation, $\mathrm{SBCR}$ ) of their components.
Based on the observation of some 35 million stars in the LMC for more than $20 \mathrm{yr}$ during the Optical Gravitational Lensing Experiment (OGLE; Udalski et al. 2008), a few dozen extremely scarce long-period eclipsing systems composed of late-type clump giants were cataloged (Graczyk et al. 2011; Pawlak et al. 2016). In the course of the Araucaria project (Gieren et al. 2005), Pietrzyński et al. (2013) studied 8 systems and derived a distance to the LMC at the $2.2 \%$ level. This distance relied on the SBCR derived by Di Benedetto (2005) and thus was completely dominated by the error of $2 \%$ (or 0.03 magnitude) on this relation. Then, Laney et al. (2012) secured uniform and precise $K$-band photometry (precision of 
Table 1. Summary of the observing log.

\begin{tabular}{llll}
\hline \hline Star & Telescope configurations & $N$ & Reference stars \\
\hline HD 11037 & E1E2W2, W1W2S2 & 4 & C1, C2 \\
HD 13468 & E1E2W2, W1W2E2 & 7 & C3 \\
HD 22798 & E1E2W2, W1W2S2 & 4 & C4 \\
HD 23526 & E1E2W2, W1W2S2 & 9 & C4 \\
HD 360 & S1S2W2 & 7 & C5, C6 \\
HD 40020 & E1E2, W1W2 & 6 & C7, C8 \\
HD 43023 & E1E2W2, W1W2S2 & 7 & C9, C10, C11, C12 \\
HD 5268 & W2W1S2 & 2 & C5, C6 \\
\hline
\end{tabular}

Notes. All the details are given in Table B.1. $N$ corresponds to the number of visibility measurements for each star. The reference stars are also indicated (cf. Table 2).

about $\simeq 0.01 \mathrm{mag}$ ) for a large sample of nearby very bright red giants located in a very well defined and quiet evolutionary phase of the core-helium burning (red clump). In addition, Gallenne et al. (2018) secured observations of 48 of these red-clump stars in the $\mathrm{H}$ band with the Precision Integrated Optics Nearinfrared Imaging ExpeRiment (PIONIER, Le Bouquin et al. 2011) installed at the Very Large Telescope Interferometer. These measurements allowed deriving an SBCR with an rms of 0.018 magnitude. This relation was used to derive the individual angular diameters of the 20 eclipsing binaries at the $0.8 \%$ precision level, which finally led to a distance of the LMC that is accurate to $1 \%$ (Pietrzyński et al. 2019).

The purpose of this paper is to calibrate the SBCR in the visible domain in order to compare it to the one derived in the $H$ band. For this, we observed a subsample of eight stars in the visible domain with interferometry, using the Visible spEctroGraph and polArimeter (VEGA) beam combiner (Mourard et al. 2009, 2011) operating at the focus of The Center for High Angular Resolution Astronomy (CHARA) array (ten Brummelaar et al. 2005), which is located at the Mount Wilson Observatory. In Sect. 2 we describe the VEGA/CHARA interferometric observations and provide the limb-darkened angular diameters of the eight stars in our sample. Section 3 is dedicated to the calibration of the SBCR. The results are then discussed in Sect. 4, and we conclude in Sect. 5.

\section{VEGA/CHARA observations of eight late-type stars}

We selected eight late-type stars with existing infrared photometry by Laney et al. (2012), with a $(V-K)_{0}$ color index ranging from 2.12 to 2.43 , which corresponds to the index that was used to derive the distance to the LMC (2.05-2.75). They are red giants stars $\left(\delta\right.$ between $-9^{\circ}$ to $\left.12^{\circ}\right)$ with spectral types ranging from G8 to $\mathrm{K} 2$. They have a visual magnitude $m_{\mathrm{V}}$ ranging from 5.8 to 6.2 , which is well below the limiting magnitude of VEGA. They are also bright in the $\mathrm{K}$ band (with $m_{\mathrm{K}}<3.9$ ), which allows us to track the group delay simultaneously with the infrared CLIMB combiner (Sturmann et al. 2010). We observed our sample from 2013 July to 2014 October using different suitable telescopes available on the CHARA array. A summary of the observations is given in Table 1 .

For these observations, we used the medium spectral resolution mode of VEGA $(R \simeq 5000)$ and the standard VEGA pipeline in order to calibrate the squared visibilities in spectral bands of 15 or $20 \mathrm{~nm}$ (Mourard et al. 2009, 2011; Ligi et al. 2013).
Table 2. Reference stars and their parameters, including the spectral type, the visual magnitude $\left(m_{\mathrm{V}}\right)$, and the predicted uniform-disk angular diameter (in mas) together with its corresponding uncertainty derived from the JMMC SearchCal software (Bonneau et al. 2006; Lafrasse et al. 2010).

\begin{tabular}{llllc}
\hline \hline & $\begin{array}{l}\text { Reference } \\
\text { stars }\end{array}$ & $\begin{array}{l}\text { Spectral } \\
\text { type }\end{array}$ & $m_{\mathrm{V}}$ & $\theta_{\mathrm{UD}}[R]$ \\
& & & {$[\mathrm{mag}]$} & {$[\mathrm{mas}]$} \\
\hline C1 & HD 18604 & B6III & 4.70 & $0.259 \pm 0.018$ \\
C2 & HD 224926 & B7III-IV & 5.10 & $0.192 \pm 0.014$ \\
C3 & HD 15633 & A3V & 6.01 & $0.252 \pm 0.018$ \\
C4 & HD 23363 & B7IV & 5.25 & $0.201 \pm 0.014$ \\
C5 & HD 219402 & A2V & 5.55 & $0.249 \pm 0.018$ \\
C6 & HD 6530 & A0V & 5.58 & $0.234 \pm 0.017$ \\
C7 & HD 34203 & A0V & 5.52 & $0.221 \pm 0.016$ \\
C8 & HD 30034 & F0V & 5.38 & $0.407 \pm 0.029$ \\
C9 & HD 43445 & B9V & 5.00 & $0.239 \pm 0.017$ \\
C10 & HD 32249 & B3IV & 4.81 & $0.176 \pm 0.012$ \\
C11 & HD 46487 & B5IV/V & 5.08 & $0.180 \pm 0.013$ \\
C12 & HD 34863 & B7/8V & 5.28 & $0.181 \pm 0.013$ \\
\hline
\end{tabular}

Notes. The uncertainties on the reference stars are about $7 \%$, which is conservative.

The integration time of our observations is of $500 \mathrm{~s}$. For each calibrated visibility, the statistical and systematic calibration errors are given separately in Table B.1. The systematic uncertainty stems from the uncertainty on the calibrator diameter, which is given in Table 2. To perform the model fitting, we used a JMMC ${ }^{1}$ tool, LITpro (Tallon-Bosc et al. 2008). The systematic and statistical errors are considered separately in the fitting procedure and are propagated until the final error on the surface brightness. In Figs. A.1 and A.2, the calibrated visibilities are plotted as a function of the spatial frequency together with the corresponding $(u, v)$ coverage for each star. We fit these calibrated visibilities by a uniform disk, where the so-called uniform-disk angular diameter $\left(\theta_{\mathrm{UD}}\right)$ is the only parameter. The results are given in Table 3 . We then converted these $\theta_{\mathrm{UD}}$ diameters into limb-darkened $\left(\theta_{\mathrm{LD}}\right)$ angular diameters. A common and convenient approach is to use a linear law for the continuum-intensity profile of the star, defined by $I(\cos (\alpha))=1-u+u \cos (\alpha)$, where $u$ is the linear limb-darkening coefficient of the star in the interferometric wavelength band of observation (Claret \& Bloemen 2011). $\alpha$ is the angle between the normal of the star and the line of sight. The limb-darkened angular diameter is then calculated using $\theta_{\mathrm{LD}}=\theta_{\mathrm{UD}}\left[\frac{\left(1-\frac{u}{3}\right)}{\left(1-\frac{7 u}{15}\right)}\right]^{\frac{1}{2}}$ (Hanbury Brown et al. 1974). In the Claret tables, $u$ is given as a function of the effective temperature $T_{\text {eff }}$, the surface gravity $\log g$, the metallicity $Z$ and the microturbulence velocity $V_{\mathrm{t}}$, in several photometric bands, including the $R$ band $\left(\lambda_{\mathrm{eff}}=670 \mathrm{~nm}\right)$ and the $I$ band $\left(\lambda_{\mathrm{eff}}=\right.$ $856 \mathrm{~nm})$. The fundamental parameters of the stars are given in Table 4 together with the rounded values used as an input in the Claret tables. After they are extracted from these tables, the limb-darkened coefficients in the $R$ and $I$ bands were interpolated at the typical wavelength band of our observations, that is, $710 \mathrm{mn}$, in order to derive the corresponding linear limbdarkening coefficient $\left(u_{[710]}\right)$. The resulting $u_{[710]}$ parameters are given in Table 3 together with the corresponding limb-darkened angular diameters. The reduced $\chi^{2}$ ranges from 0.4 to 2.9 .

1 http://www.jmmc.fr/ 
N. Nardetto et al.: Calibrating the surface brightness - color relation for late-type stars

Table 3. Angular diameters obtained with VEGA/CHARA and the corresponding surface brightnesses.

\begin{tabular}{|c|c|c|c|c|c|c|c|c|c|c|}
\hline Star & $\begin{array}{c}m_{\mathrm{V}} \\
{[\mathrm{mag}]}\end{array}$ & $\begin{array}{c}m_{\mathrm{K}} \\
{[\mathrm{mag}]}\end{array}$ & $\begin{array}{c}A_{\mathrm{V}} \\
{[\mathrm{mag}]}\end{array}$ & $\begin{array}{c}(V-K)_{0} \\
{[\mathrm{mag}]}\end{array}$ & $\begin{array}{c}\theta_{\mathrm{UD}}^{ \pm \sigma_{\text {stat }}} \\
{[\mathrm{mas}]}\end{array}$ & $\chi_{\text {red }}^{2}$ & $u_{[710]}$ & $\begin{array}{c}\theta_{\mathrm{LD}}^{ \pm \sigma_{\text {stat }}} \\
{[\mathrm{mas}]}\end{array}$ & $\begin{array}{c}S_{\mathrm{v} \pm \sigma_{\text {stat }}}^{ \pm \sigma_{\text {syt }}} \\
{[\mathrm{mag}]}\end{array}$ & $\begin{array}{c}\theta_{\text {LDPIONIER }}^{ \pm \sigma_{\text {stat }}} \\
{[\mathrm{mas}]}\end{array}$ \\
\hline HD 11037 & $5.910_{ \pm 0.010}$ & $3.666_{ \pm 0.010}$ & 0.031 & 2.216 & $0.841_{ \pm 0.002}^{ \pm 0.018}$ & 1.3 & 0.631 & $0.890_{ \pm 0.002}^{ \pm 0.019}$ & $5.626_{ \pm 0.011}^{ \pm 0.047}$ & \multirow{3}{*}{$0.886^{ \pm 0.009}$} \\
\hline HD $13468^{*}$ & $5.940_{ \pm 0.010}$ & $3.666_{ \pm 0.010}$ & 0.028 & 2.248 & $0.851_{ \pm 0.002}^{ \pm 0.016}$ & 1.6 & 0.628 & $0.900_{ \pm 0.002}^{ \pm 0.017}$ & $5.683_{ \pm 0.011}^{ \pm 0.042}$ & \\
\hline HD 22798 & $6.220_{ \pm 0.010}$ & $3.880_{ \pm 0.010}$ & 0.012 & 2.329 & $0.749_{ \pm 0.002}^{ \pm 0.020}$ & 2.9 & 0.631 & $0.792_{ \pm 0.002}^{ \pm 0.021}$ & $5.701_{ \pm 0.011}^{ \pm 0.058}$ & \\
\hline HD $23526^{*}$ & $5.910_{ \pm 0.010}$ & $3.634_{ \pm 0.010}$ & 0.053 & 2.228 & $0.869_{ \pm 0.001}^{ \pm 0.0025}$ & 0.6 & 0.631 & $0.920_{ \pm 0.001}^{ \pm 0.027}$ & $5.676_{ \pm 0.010}^{ \pm 0.064}$ & \multirow{4}{*}{$\begin{array}{l}0.915^{ \pm 0.020} \\
0.906^{ \pm 0.014} \\
1.012^{ \pm 0.022}\end{array}$} \\
\hline HD $360^{*}$ & $5.990_{ \pm 0.010}$ & $3.653_{ \pm 0.010}$ & 0.028 & 2.311 & $0.835_{ \pm 0.002}^{ \pm 0.009}$ & 0.5 & 0.658 & $0.886_{ \pm 0.002}^{ \pm 0.010}$ & $5.699_{ \pm 0.011}^{ \pm 0.026}$ & \\
\hline $\begin{array}{l}\text { HD } 40020^{*} \\
\text { HD } 43023\end{array}$ & $\begin{array}{l}5.890_{ \pm 0.010} \\
5.830_{ \pm 0.010}\end{array}$ & $\begin{array}{l}3.419_{ \pm 0.010} \\
3.704_{ \pm 0.010}\end{array}$ & $\begin{array}{l}0.040 \\
0.003\end{array}$ & $\begin{array}{l}2.434 \\
2.123\end{array}$ & $\begin{array}{l}0.969_{ \pm 0.005}^{ \pm 0.024} \\
0.796_{ \pm 0.001}^{ \pm 0.0102}\end{array}$ & $\begin{array}{l}0.4 \\
1.1\end{array}$ & $\begin{array}{l}0.668 \\
0.631\end{array}$ & $\begin{array}{l}1.030_{ \pm 0.005}^{ \pm 0.025} \\
0.842_{ \pm 0.001}^{ \pm 0.014}\end{array}$ & $\begin{array}{l}5.913^{ \pm 0.0053} \\
5.453_{ \pm 0.014}^{ \pm 0.037}\end{array}$ & \\
\hline HD 5268 & $6.150_{ \pm 0.010}$ & $3.910_{ \pm 0.010}$ & 0.043 & 2.200 & $0.725_{ \pm 0.005}^{ \pm 0.033}$ & 0.5 & 0.628 & $0.767_{ \pm 0.005}^{ \pm 0.035}$ & $5.530_{ \pm 0.017}^{ \pm 0.099}$ & \\
\hline
\end{tabular}

Notes. For each star, we consider an uncertainty of 0.01 magnitude for $m_{\mathrm{V}}$ and $m_{\mathrm{K}}$ (Laney et al. 2012). The angular diameter of stars marked with an asterisk is based on PIONIER/VLTI observations provided in the extended data Table 2 of Pietrzyński et al. (2019). This is reported here in the last column.

Table 4. Fundamental parameters of the stars in our sample based on spectroscopy and combined with photometry for stars in Luck \& Heiter (2007).

\begin{tabular}{lcccccccccc}
\hline \hline Star & Sp. type & $T_{\text {eff }}$ & $\log g$ & $\mathrm{Z}$ & $V_{t}$ & $\begin{array}{c}\text { Round } \\
T_{\text {eff }} \\
{[\mathrm{K}]}\end{array}$ & $\begin{array}{c}\text { Rround } \\
\log g\end{array}$ & $\begin{array}{c}\text { Round } \\
\mathrm{Z}\end{array}$ & $\begin{array}{c}\text { Round } \\
V_{t} \\
{\left[\mathrm{~km} \mathrm{~s}^{-1}\right]}\end{array}$ & References \\
\hline HD 11037 & K0III & 4976 & 2.85 & -0.10 & 1.5 & 5000 & 3.0 & 0.0 & 1.0 & Luck \& Heiter (2007) \\
HD 13468 & K0III & 4940 & 2.59 & -0.17 & 1.0 & 5000 & 2.5 & 0.0 & 1.0 & Jones et al. (2011) \\
HD 22798 & K0III & 4905 & 2.99 & 0.24 & 1.0 & 5000 & 3.0 & 0.2 & 1.0 & Soubiran et al. (2008) \\
HD 23526 & G9III & 4935 & 2.81 & -0.12 & 1.0 & 5000 & 3.0 & 0.0 & 1.0 & Luck \& Heiter (2007) \\
HD 360 & G9III & 4741 & 2.73 & -0.05 & 1.3 & 4750 & 3.0 & 0.0 & 1.0 & Liu et al. (2007) \\
HD 40020 & K2III & 4752 & 2.67 & 0.17 & 2.0 & 4750 & 2.5 & 0.2 & 2.0 & Luck \& Heiter (2007) \\
HD 43023 & K0III & 5105 & 3.08 & -0.06 & 1.5 & 5000 & 3.0 & 0.0 & 1.0 & Luck \& Heiter (2007) \\
HD 5268 & G8III & 4904 & 2.35 & -0.57 & 1.0 & 5000 & 2.5 & 0.0 & 1.0 & Jones et al. (2011) \\
\hline
\end{tabular}

Changing the effective temperature by $500 \mathrm{~K}$ and/or the surface gravity by 0.5 have an effect on the limb-darkening angular diameter of $1.3 \%$. The grid in effective temperature has a step of $250 \mathrm{~K}$, thus the largest error we can make on the temperature is $125 \mathrm{~K}$, which corresponds to an additional uncertainty on the limb-darkened diameter of about $0.3 \%$. However, the fact that the derived diameters are weakly sensitive to the input parameters of the limb-darkening law does not exclude that they might be sensitive to the limb-darkening law itself or to the method. We also tested the method by fitting the limb-darkened angular diameter with the limb_linear function of the LITpro tool, that is, by fixing the limb-darkening coefficient to its value indicated in Table 4. The derived limb-darkened angular diameters are 0.4 to $0.8 \%$ larger (except for HD 23526, for which we find 1.2\%) than those indicated in Table 4 based on the approximate analytic conversion law. This bias is larger than what has been stated by Hanbury Brown et al. (1974), which is an approximation of $<0.4 \%$ if $u_{\mathrm{R}}$ ranges from 0.5 to 1.0 . The uncertainties are strictly the same, however. For the limb-darkening law itself, we also used the dedicated SATLAS models by Neilson \& Lester $(2013 \mathrm{a}, \mathrm{b})$ to derive the limb-darkened diameters using the same input physical parameters as those indicated in Table 4 . With this approach, we find diameters that are about $0.4 \%$ larger than the values presented in Table 3. These tests are interesting and should be considered with caution when a limb-darkened angular diameter is derived at the sub-percent level, but our statistical precision here is about $2.4 \%$ and the common analytical approach leads to a bias on our SBCR calibration at a far lower level than our statistical precision.

Our sample includes four stars that are in common with samples of Gallenne et al. (2018) and Pietrzyński et al. (2019): HD13468, HD 23526, HD 360, and HD 40020. Their PIONIER/VLTI angular diameters are listed in Table 3. This is an interesting opportunity to compare the angular diameters derived from CHARA/VEGA and VLTI/PIONIER (see Fig. 1). We find that the CHARA/VEGA angular diameters are consistent within their uncertainties with those from VLTI/PIONIER. Visible (this work) and infrared (Gallenne et al. 2018) interferometric observations are thus in agreement, which means that the conversions from UD into LD angular diameters are at least consistent in the two wavelength domains $(R$ and $H$ ) at the sub-percent level. Moreover, it indicates that there is negligible systematics on the instrumental point of view in the determination of the angular diameters. Both instruments might be biased in exactly the same way (for the four stars), but this is highly unlikely. The calibrators used in the VLTI/PIONIER analysis are giants with spectral types ranging from G8 to $\mathrm{K} 2$, which is significantly colder and more resolved than the calibrators we used in our analysis in the visible domain (Table 2). We therefore expect that the systematics on the squared visibilities (Table 2) that stem from 


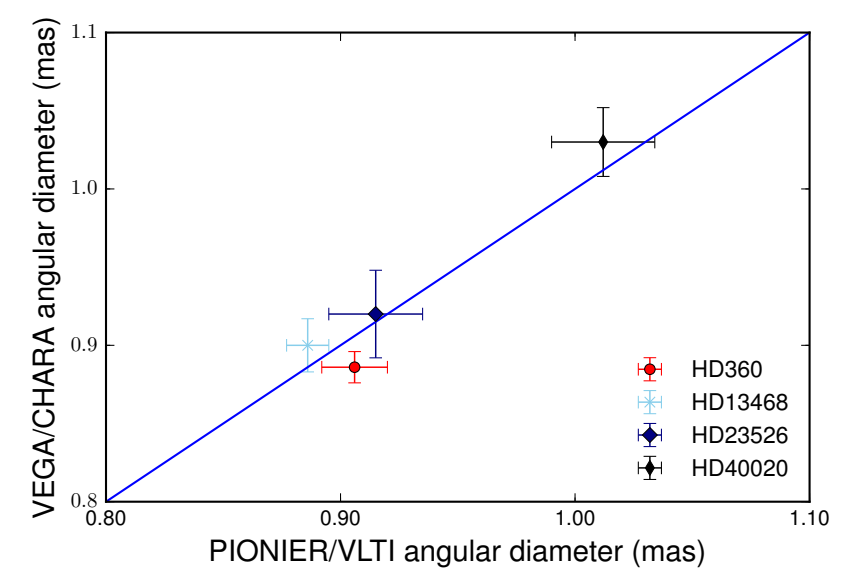

Fig. 1. Angular diameters derived from VEGA/CHARA compared to those from PIONIER/VLTI. The blue line marks the 1:1 relation.

the uncertainties on the calibrators are significantly lower in the visible than in the infrared.

\section{Calibrating the surface brightness relation}

The surface brightness of a star is linked to its visual intrinsic dereddened magnitude $m_{\mathrm{V}_{0}}$ and its limb-darkened angular diameter $\theta_{\mathrm{LD}}$ by the following relation: $S_{\mathrm{V}}=m_{\mathrm{V}_{0}}+5 \log \theta_{\mathrm{LD}}$. In order to derive $m_{\mathrm{V}_{0}}$, we first selected the apparent $m_{\mathrm{V}}$ magnitude from the main HIPPARCOS and Tycho catalog (ESA 1997) expressed in the Johnson system (Johnson et al. 1966). For the dereddening of these magnitudes, we used $m_{\mathrm{V}_{0}}=m_{\mathrm{V}}-A_{\mathrm{V}}$, where $A_{\mathrm{V}}$ is either taken directly from Pietrzyński et al. (2019) for the four targets we have in common or based on Stilism ${ }^{2}$ (Lallement et al. 2014; Capitanio et al. 2017). Pietrzyński et al. (2019) used the 2D reddening maps of Schlegel et al. (1998) and Schlafly \& Finkbeiner (2011). These maps give a total extinction in a given line of sight, while the reddening to a given star is only a small fraction of it. To calculate this fraction, they used a simple exponential model of dust distribution in the Milky Way, the HiPPARCOS parallaxes, and the local extinction-free bubble radius of $40 \mathrm{pc}$ (Suchomska et al. 2015). Stilism is a 3D maps of the Galactic interstellar medium based on a combination of various methods and databases. The extinction we used for each star in our sample is indicated in Table 3, and the effect of these extinctions on the SBCR is discussed in the next section. Then the color follows with $(V-K)_{0}=(V-K)-A_{\mathrm{V}}-A_{\mathrm{K}}$, where $A_{\mathrm{K}}=0.089 A_{\mathrm{V}}$ (Nishiyama et al. 2009). The $m_{\mathrm{K}}$ magnitudes are homogeneously derived from Laney et al. (2012). The $S_{\mathrm{V}}$ values are given in Table 3.

In order to fit our SBCR, we used the same formalism as Pietrzyński et al. (2019),

$S_{\mathrm{V}}=\alpha\left[(V-K)_{0}-2.405\right]+\beta$,

where $\alpha$ and $\beta$ are the slope and zero-point of the relation, respectively. The value 2.405 is the average color of the stars that were used to built the SBCR in Pietrzyński et al. (2019). We kept this value in our fit to allow a direct comparison. We first fit both parameters and then assumed the slope from the SBCR of Pietrzyński et al. (2019). The statistical uncertainty on the magnitudes $m_{\mathrm{V}}$ and $m_{\mathrm{K}}$, on the extinction $A_{\mathrm{V}}$, and on the surface brightness $S_{\mathrm{v}}$ were taking into account in the fitting process. Results are summarized in Table 5. We obtain very

2 https://stilism.obspm.fr/ similar results when we fit one or two parameters $(1.7 \%$ difference on the slope and $0.05 \%$ on the zero-point), as shown by Fig. 2. The SBCRs from Kervella et al. (2004a), Di Benedetto (2005), and Challouf et al. (2014) are shown for comparison.

\section{Discussion}

Our relation has an rms of 0.04 magnitude (Table 5) and is consistent with all other SBCRs presented in Fig. 2, except for that of Challouf et al. (2014), in particular for a $(V-K)$ color larger than 2.3 magnitude. This is explained by the fact that the relation by Challouf et al. (2014) is nonlinear, was based on 132 stars of all classes, and was fit over a very wide range of color from early to late types. We note that this relation was dedicated to $\mathrm{O}, \mathrm{B}, \mathrm{A}$ stars.

Conversely, the relation by Pietrzyński et al. (2019), which has an rms of 0.018 magnitude, is consistent with all SBCRs (except for that of Challouf et al. (2014)), including the SBCR in this work. These relations are indeed consistent (within $1 \%$ ), even though different methods were used. The relation by Pietrzyński et al. (2019) is based on the observation of 48 giant stars in the $\mathrm{H}$ band with the PIONIER/VLTI (Gallenne et al. 2018), while the relation by Kervella et al. (2004a) is based on a set of angular diameters of dwarfs and subgiant stars derived from different optical and infrared interferometers: NPOI, NII (Narrabri Intensity Interferometer, Hanbury Brown et al. 1967), Mark III (Shao et al. 1988), PTI (Palomar Testbed Interferometer, Colavita et al. 1999), and VINCI (Kervella et al. 2004b). The relation by Di Benedetto 2005 is based on the NPOI (Navy Prototype Optical Interferometer, Armstrong et al. 1998) observations by Nordgren et al. (2001) of 24 giants and three dwarfs obtained in the optical domains (wavelength of reference of $740 \mathrm{~nm}$ ). Thus, following Salsi et al. (in prep.), who found a different SBCR depending on the spectral type and class of the stars, we can consistently compare the relations by Pietrzyński et al. (2019), Di Benedetto (2005) and ours, which are all based on giants. On the color range considered, the relation in this work (rms of $0.039 \mathrm{mag}$ ) is consistent with the relation by Di Benedetto (2005) (rms of $0.04 \mathrm{mag}$ ) and with the relation by Pietrzyński et al. (2019) (rms of $0.018 \mathrm{mag}$ ).

It is well known that the extinction has a negligible effect on the SBCR because the color and surface brightness are sensitive to it in almost the same way. The only difference comes from the extinction in $\mathrm{K}$, which is about $10 \%$ of the extinction in V. Adding an extinction of 0.1 magnitude on a measurement, for instance, results in a brighter color of the star of about 0.054 magnitude, and similarly, in a brighter surface-brightness of 0.05 magnitude. In order to quantify the effect of extinction on the SBCR, we arbitrarily changed the absorption $\left(A_{\mathrm{V}}\right)$ of all stars in our sample. We find that a higher extinction of 0.1 magnitude results in an increase in zero-point of the SBCR by 0.045 magnitude, which corresponds to the rms of our SBCR. This offset becomes 0.048 magnitude when we use the older relationship $A_{\mathrm{K}}=0.114 A_{\mathrm{V}}$ from Cardelli et al. (1989) instead of the relation we considered in Sect. $3\left(A_{\mathrm{K}}=0.089 A_{\mathrm{V}}\right)$ from Nishiyama et al. (2009). This means that even if the $A_{\mathrm{V}}$ values of the star in the sample were all under-estimated by 0.1 magnitude (which is highly unlikely), our SBCR would still be consistent with the SBCR by Pietrzyński et al. (2019).

\section{Conclusion}

We observed eight red clump stars (late-type giants) with the VEGA/CHARA instrument and derived their angular diameters 


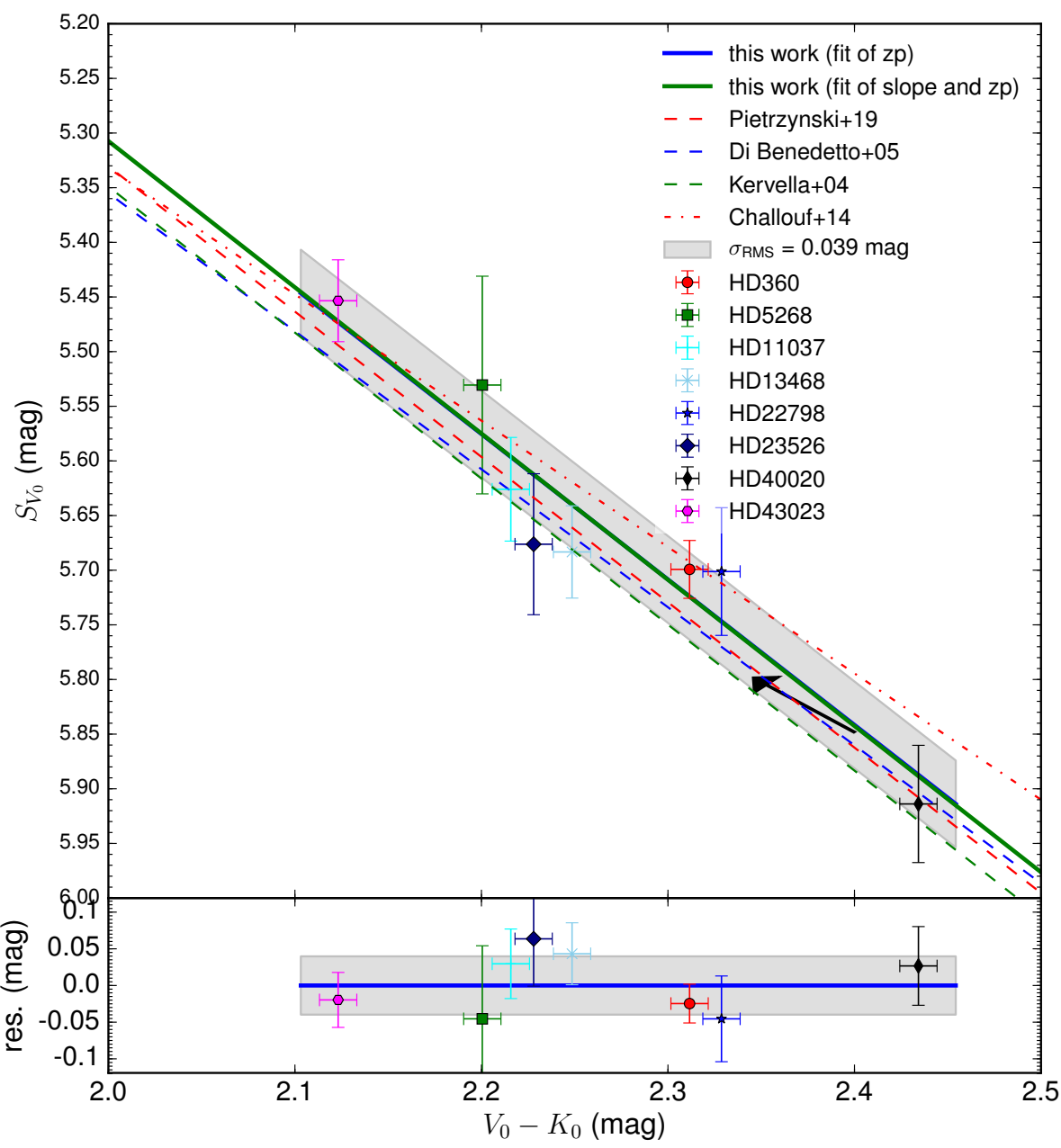

Fig. 2. Surface brightness - color relation derived from VEGA/CHARA data of eight late-type stars with a homogenous set of infrared photometry (Laney et al. 2012). The black arrow shows the effect of an additional extinction of 0.1 magnitude on a measurement. The gray zone corresponds to the rms.

Table 5. Fit of the SBCR. Slope and zero-point of the SBCR were first fit using Eq. (1).

\begin{tabular}{llll}
\hline \hline Reference & $\beta$ & $\alpha$ & rms \\
\hline Pietrzyński et al. (2019) & $5.869_{ \pm 0.003}$ & $1.330_{ \pm 0.017}$ & 0.018 \\
This work & $5.849_{ \pm 0.027}$ & $1.338_{ \pm 0.160}$ & 0.039 \\
This work & $5.848_{ \pm 0.013}$ & $1.330^{\star}$ & 0.039 \\
\hline
\end{tabular}

Notes. We then assumed the slope from the SBCR of Pietrzyński et al. (2019) (indicated by an asterisk). The reduced $\chi^{2}$ is 0.4 in both cases.

with an average precision of $2.4 \%$. By combining these diameters with a homogeneous set of infrared photometry (Laney et al. 2012), we derived the slope and zero-point of the SBCR with a residual dispersion of 0.04 magnitude (or $1.8 \%$ precision in terms of angular diameter). The zero-points of the SBCR derived in the $\mathrm{H}$ band and in the visible domain are consistent at the $0.4 \%$ level $($ or $0.5 \sigma)$. The angular diameters of four stars in our sample are consistent with those found by VLTI/PIONIER, which shows that the systematics of both instruments are under control It also indicates that it is possible to combine interferometric measurements in different bands when the SBCR is calibrated. All these results confirm the recent claim of a $1 \%$ precision and accuracy on the LMC distance by Pietrzyński et al. (2019).

Acknowledgements. We acknowledge M. Schultheis for fruitful discussions about the extinction of stars. This research has made use of the SIMBAD and VIZIER (http: //cdsweb.u-strasbg.fr/) databases at CDS, Strasbourg (France) and of electronic bibliography maintained by the NASA/ADS system. This research has made use of the Jean-Marie Mariotti Center LITpro service co-developped by CRAL, IPAG and Lagrange (LITpro software available at http://www.jmmc.fr/litpro). This research has made use of the JeanMarie Mariotti Center Aspro service (http://www.jmmc.fr/aspro). This work was supported by the "Programme National de Physique Stellaire (PNPS) of CNRS/INSU co-funded by CEA and CNES". This work was supported by the "Action Spécifique pour la Haute Résolution Angulaire (ASHRA) of CNRS/INSU co-funded by CNES". The CHARA Array is funded by the National Science Foundation through NSF grants AST-0606958 and AST0908253 and by Georgia State University through the College of Arts and Sciences, as well as the W. M. Keck Foundation. R.L. has received funding from the European Union's Horizon 2020 research and innovation programme under the Marie Sklodowska-Curie grant agreement n. 664931. W.G. and G.P. gratefully acknowledge financial support for this work from the BASAL Centro de Astrofisica y Tecnologias Afines (CATA) AFB-170002. We acknowledge financial support for this work from ECOS-CONICYT grant C13U01. The authors acknowledge the support of the FrenchAgence Nationale de la Recherche (ANR), under grant ANR-15-CE31-0012-01 (project UnlockCepheids). Support from the Polish National Science Centre grants MAESTRO UMO-2017/26/A/ST9/00446 and from the IdPII 2015000264 grant of the Polish Ministry of Science and Higher Education is also acknowl-edged. The research leading to these results has received funding from the European Re-search Council (ERC) under the European Unions Horizon 2020 research and innovation program (grant agreement No 695099). 


\section{References}

Armstrong, J. T., Mozurkewich, D., Rickard, L. J., et al. 1998, ApJ, 496, 550

Bonneau, D., Clausse, J.-M., Delfosse, X., et al. 2006, A\&A, 456, 789

Capitanio, L., Lallement, R., Vergely, J. L., Elyajouri, M., \& Monreal-Ibero, A. 2017, A\&A, 606, A65

Cardelli, J. A., Clayton, G. C., \& Mathis, J. S. 1989, ApJ, 345, 245

Challouf, M., Nardetto, N., Mourard, D., et al. 2014, A\&A, 570, A104

Claret, A., \& Bloemen, S. 2011, A\&A, 529, A75

Colavita, M. M., Wallace, J. K., Hines, B. E., et al. 1999, ApJ, 510, 505

Di Benedetto, G. P. 2005, MNRAS, 357, 174

ESA 1997, ESA Special Publication, 1200, The HIPPARCOS and TYCHO catalogues. Astrometric and photometric star catalogues derived from the ESA HIPPARCOS Space Astrometry Mission

Freedman, W. L., \& Madore, B. F. 2010, ApJ, 719, 335

Gallenne, A., Pietrzyński, G., Graczyk, D., et al. 2018, A\&A, 616, A68

Gieren, W., Storm, J., Barnes, III, T. G., et al. 2005, ApJ, 627, 224

Graczyk, D., Soszyński, I., Poleski, R., et al. 2011, Acta Astron., 61, 103

Hanbury Brown, R., Davis, J., \& Allen, L. R. 1967, MNRAS, 137, 375

Hanbury Brown, R., Davis, J., Lake, R. J. W., \& Thompson, R. J. 1974, MNRAS 167,475

Johnson, H. L., Mitchell, R. I., Iriarte, B., \& Wisniewski, W. Z. 1966, Commun. Lunar Planet. Lab., 4, 99

Jones, M. I., Jenkins, J. S., Rojo, P., \& Melo, C. H. F. 2011, A\&A, 536, A71

Kervella, P., Thévenin, F., Di Folco, E., \& Ségransan, D. 2004a, A\&A, 426, 297

Kervella, P., Ségransan, D., \& Coudé du Foresto, V. 2004b, A\&A, 425, 1161

Komatsu, E., Smith, K. M., Dunkley, J., et al. 2011, ApJS, 192, 18

Lafrasse, S., Mella, G., Bonneau, D., et al. 2010, VizieR Online Data Catalog. II/ $/ 300$

Lallement, R., Vergely, J.-L., Valette, B., et al. 2014, A\&A, 561, A91

Laney, C. D., Joner, M. D., \& Pietrzyński, G. 2012, MNRAS, 419, 1637

Le Bouquin, J.-B., Berger, J.-P., Lazareff, B., et al. 2011, A\&A, 535, A67
Ligi, R., Mourard, D., Nardetto, N., \& Clausse, J.-M. 2013, J. Astron. Instrum., 2, 40003

Liu, Y. J., Zhao, G., Shi, J. R., Pietrzyński, G., \& Gieren, W. 2007, MNRAS, 382,553

Luck, R. E., \& Heiter, U. 2007, AJ, 133, 2464

Mourard, D., Clausse, J. M., Marcotto, A., et al. 2009, A\&A, 508, 1073

Mourard, D., Bério, P., Perraut, K., et al. 2011, A\&A, 531, A110

Neilson, H. R., \& Lester, J. B. 2013a, A\&A, 554, A98

Neilson, H. R., \& Lester, J. B. 2013b, A\&A, 556, A86

Nishiyama, S., Tamura, M., Hatano, H., et al. 2009, ApJ, 696, 1407

Nordgren, T. E., Sudol, J. J., \& Mozurkewich, D. 2001, AJ, 122, 2707

Pawlak, M., Soszyński, I., Udalski, A., et al. 2016, Acta Astron., 66, 421

Pietrzyński, G., Graczyk, D., Gieren, W., et al. 2013, Nature, 495, 76

Pietrzyński, G., Graczyk, D., Gallenne, A., et al. 2019, Nature, 567, 200

Riess, A. G., Macri, L. M., Hoffmann, S. L., et al. 2016, ApJ, 826, 56

Riess, A. G., Casertano, S., Yuan, W., Macri, L. M., \& Scolnic, D. 2019, ApJ, 876,85

Schaefer, B. E. 2008, AJ, 135, 112

Schlafly, E. F., \& Finkbeiner, D. P. 2011, ApJ, 737, 103

Schlegel, D. J., Finkbeiner, D. P., \& Davis, M. 1998, ApJ, 500, 525

Shao, M., Colavita, M. M., Hines, B. E., et al. 1988, ApJ, 327, 905

Soubiran, C., Bienaymé, O., Mishenina, T. V., \& Kovtyukh, V. V. 2008, A\&A, 480,91

Sturmann, J., ten Brummelaar, T., Sturmann, L., \& McAlister, H. A. 2010, SPIE, Conf. Ser., 7734, 3

Suchomska, K., Graczyk, D., Smolec, R., et al. 2015, MNRAS, 451, 651

Tallon-Bosc, I., Tallon, M., Thiébaut, E., et al. 2008, SPIE Conf. Ser., 7013, $70131 \mathrm{~J}$

ten Brummelaar, T. A., McAlister, H. A., Ridgway, S. T., et al. 2005, ApJ, 628, 453

Udalski, A., Szymanski, M. K., Soszynski, I., \& Poleski, R. 2008, Acta Astron., 58,69

Walker, E. S., Hachinger, S., Mazzali, P. A., et al. 2012, MNRAS, 427, 103 
Appendix A: Plot of the CHARA/VEGA squared visibility measurements
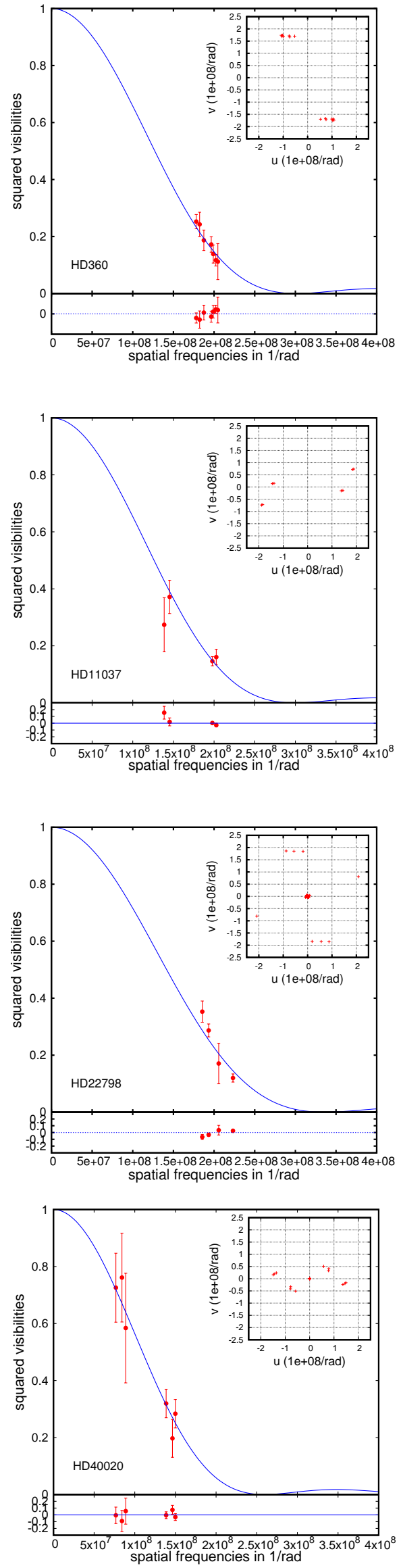
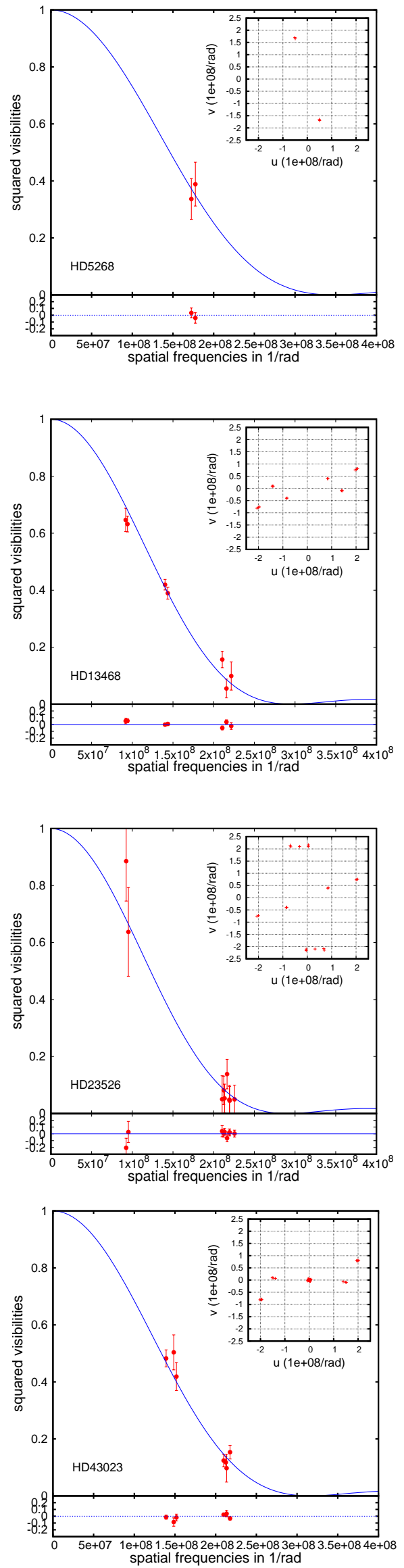

Fig. A.1. Squared visibility vs. spatial frequency for all stars in our sample with their corresponding statistical uncertainties (red dots). The solid blue lines indicate the best uniformdisk model obtained from the LITpro fitting software.

Fig. A.2. Squared visibility vs. spatial frequency for all stars in our sample with their corresponding statistical uncertainties (red dots). The solid blue lines indicate the best uniform-disk model obtained from the LITpro fitting software. 


\section{Appendix B: Table of the observing log}

Table B.1. Observing log.

\begin{tabular}{|c|c|c|c|c|c|c|c|c|c|}
\hline & $\begin{array}{c}\text { Date } \\
\text { [yyyy.mm.dd] }\end{array}$ & $\begin{array}{c}\text { RJD } \\
\text { [days] }\end{array}$ & $\begin{array}{l}\mathrm{HA} \\
{[\mathrm{h}]}\end{array}$ & $\begin{array}{l}\lambda_{\min } \\
{[\mathrm{nm}]}\end{array}$ & $\begin{array}{l}\lambda_{\max } \\
{[\mathrm{nm}]}\end{array}$ & Baseline & $\begin{array}{l}\mathrm{Bp} \\
{[\mathrm{m}]}\end{array}$ & $\begin{array}{c}\text { PA } \\
{[\mathrm{deg}]}\end{array}$ & $V_{\mathrm{cal} \pm \mathrm{stat} \pm \mathrm{syst}}^{2}$ \\
\hline \multirow[t]{4}{*}{ HD 11037} & 2013.10 .27 & 56592.686 & -2.60 & 705 & 725 & E2W2 & 144.95 & -111.33 & $0.160_{ \pm 0.027 \pm 0.003}$ \\
\hline & 2013.10 .27 & 56592.686 & -2.60 & 725 & 740 & E2W2 & 144.95 & -111.33 & $0.146_{ \pm 0.016 \pm 0.002}$ \\
\hline & 2014.10.19 & 56949.773 & -1.03 & 695 & 715 & W1W2 & 97.74 & -83.73 & $0.274_{ \pm 0.095 \pm 0.002}$ \\
\hline & 2014.10.19 & 56949.793 & -0.57 & 695 & 715 & W1W2 & 102.47 & -84.45 & $0.372_{ \pm 0.058 \pm 0.003}$ \\
\hline \multirow[t]{7}{*}{ HD 13468} & 2014.08 .27 & 56896.906 & -1.66 & 690 & 710 & E1E2 & 65.85 & -115.41 & $0.632_{ \pm 0.027 \pm 0.003}$ \\
\hline & 2014.08 .27 & 56896.906 & -1.66 & 690 & 710 & E2W2 & 155.13 & -111.55 & $0.099 \pm 0.050 \pm 0.003$ \\
\hline & 2014.08.27 & 56896.945 & -0.65 & 690 & 710 & E1E2 & 64.31 & -115.57 & $0.647 \pm 0.041 \pm 0.003$ \\
\hline & 2013.11.02 & 56598.777 & -0.51 & 705 & 725 & W1W2 & 102.72 & 93.72 & $0.389_{ \pm 0.021 \pm 0.004}$ \\
\hline & 2013.11.02 & 56598.777 & -0.51 & 705 & 725 & W2E2 & 154.24 & 68.88 & $0.055 \pm 0.033 \pm 0.001$ \\
\hline & 2013.11.02 & 56598.777 & -0.51 & 725 & 740 & W1W2 & 102.72 & 93.72 & $0.419_{ \pm 0.019 \pm 0.004}$ \\
\hline & 2013.11.02 & 56598.777 & -0.51 & 725 & 740 & W2E2 & 154.24 & 68.88 & $0.157_{ \pm 0.029 \pm 0.004}$ \\
\hline \multirow[t]{4}{*}{ HD 22798} & 2014.08 .22 & 56892.002 & -1.24 & 690 & 710 & E2W2 & 156.25 & -111.20 & $0.120_{ \pm 0.014 \pm 0.002}$ \\
\hline & 2014.10 .20 & 56950.816 & -1.83 & 695 & 715 & $\mathrm{~W} 2 \mathrm{~S} 2$ & 130.75 & -5.76 & $0.353_{ \pm 0.037 \pm 0.004}$ \\
\hline & 2014.10 .20 & 56950.853 & -0.91 & 695 & 715 & $\mathrm{~W} 2 \mathrm{~S} 2$ & 136.30 & -16.83 & $0.287_{ \pm 0.023 \pm 0.004}$ \\
\hline & 2014.10 .20 & 56950.890 & -0.06 & 695 & 715 & W2S2 & 145.01 & -25.27 & $0.171_{ \pm 0.071 \pm 0.002}$ \\
\hline \multirow[t]{9}{*}{ HD 23526} & 2014.08 .22 & 56891.974 & -1.96 & 680 & 700 & E2W2 & 151.53 & -110.15 & $0.045_{ \pm 0.050 \pm 0.001}$ \\
\hline & 2014.08 .22 & 56891.974 & -1.96 & 700 & 720 & E2W2 & 151.53 & -110.15 & $0.053_{ \pm 0.050 \pm 0.001}$ \\
\hline & 2014.08 .23 & 56892.985 & -1.64 & 680 & 700 & E1E2 & 65.66 & 64.98 & $0.637_{ \pm 0.156 \pm 0.002}$ \\
\hline & 2014.08 .23 & 56892.985 & -1.64 & 700 & 720 & E1E2 & 65.66 & 64.98 & $0.886_{ \pm 0.140 \pm 0.002}$ \\
\hline & 2014.10 .20 & 56950.797 & -2.38 & 680 & 700 & $\mathrm{~W} 2 \mathrm{~S} 2$ & 149.38 & -178.82 & $0.138_{ \pm 0.052 \pm 0.002}$ \\
\hline & 2014.10 .20 & 56950.797 & -2.38 & 700 & 720 & W2S2 & 149.38 & -178.82 & $0.050_{ \pm 0.082 \pm 0.001}$ \\
\hline & 2014.10 .20 & 56950.834 & -1.51 & 700 & 720 & $\mathrm{~W} 2 \mathrm{~S} 2$ & 150.78 & 171.40 & $0.081_{ \pm 0.050 \pm 0.001}$ \\
\hline & 2014.10 .20 & 56950.872 & 0.59 & 680 & 700 & $\mathrm{~W} 2 \mathrm{~S} 2$ & 155.72 & 161.98 & $0.050_{ \pm 0.050 \pm 0.001}$ \\
\hline & 2014.10 .20 & 56950.872 & 0.59 & 700 & 720 & W2S2 & 155.72 & 161.98 & $0.049_{ \pm 0.050 \pm 0.001}$ \\
\hline \multirow[t]{7}{*}{ HD 360} & 2013.08 .26 & 56530.922 & 0.55 & 695 & 715 & W2S2 & 144.26 & -31.84 & $0.112_{ \pm 0.063 \pm 0.002}$ \\
\hline & 2013.08.26 & 56530.922 & 0.55 & 715 & 735 & $\mathrm{~W} 2 \mathrm{~S} 2$ & 144.26 & -31.84 & $0.138_{ \pm 0.032 \pm 0.002}$ \\
\hline & 2013.07.24 & 56497.945 & -1.00 & 690 & 710 & W2S2 & 124.66 & -17.34 & $0.252 \pm 0.025 \pm 0.006$ \\
\hline & 2013.07.24 & 56497.971 & -0.41 & 690 & 710 & $\mathrm{~W} 2 \mathrm{~S} 2$ & 131.20 & -23.79 & $0.187_{ \pm 0.036 \pm 0.004}$ \\
\hline & 2013.07 .24 & 56497.971 & -0.41 & 710 & 730 & W2S2 & 131.20 & -23.79 & $0.243_{ \pm 0.043 \pm 0.005}$ \\
\hline & 2013.07.24 & 56498.003 & 0.35 & 690 & 710 & W2S2 & 141.49 & -30.45 & $0.117_{ \pm 0.020 \pm 0.003}$ \\
\hline & 2013.07.24 & 56498.003 & 0.35 & 710 & 730 & W2S2 & 141.49 & -30.45 & $0.172 \pm 0.027 \pm 0.005$ \\
\hline \multirow[t]{6}{*}{ HD40020 } & 2013.10 .27 & 56592.860 & -2.63 & 725 & 745 & E1E2 & 61.90 & 67.00 & $0.761_{ \pm 0.156 \pm 0.002}$ \\
\hline & 2013.11 .26 & 56622.863 & 0.59 & 725 & 745 & E1E2 & 65.35 & 62.21 & $0.584_{ \pm 0.192 \pm 0.006}$ \\
\hline & 2013.11 .26 & 56622.955 & 1.57 & 705 & 725 & E1E2 & 54.87 & 48.71 & $0.726_{ \pm 0.121 \pm 0.008}$ \\
\hline & 2014.10 .22 & 56952.938 & -1.05 & 700 & 720 & W1W2 & 98.39 & -80.11 & $0.319_{ \pm 0.050 \pm 0.010}$ \\
\hline & 2014.10 .22 & 56952.962 & 0.45 & 700 & 720 & W1W2 & 103.90 & -82.36 & $0.197 \pm 0.067 \pm 0.006$ \\
\hline & 2014.10 .23 & 56953.976 & 0.04 & 700 & 720 & W1W2 & 106.40 & -83.77 & $0.284_{ \pm 0.050 \pm 0.007}$ \\
\hline \multirow[t]{7}{*}{ HD43023 } & 2013.10 .26 & 56591.903 & -1.98 & 710 & 725 & E2W2 & 153.65 & -112.31 & $0.097 \pm 0.049 \pm 0.001$ \\
\hline & 2013.10 .26 & 56591.903 & -1.98 & 725 & 740 & E2W2 & 153.65 & -112.31 & $0.124_{ \pm 0.022 \pm 0.001}$ \\
\hline & 2013.10 .26 & 56591.919 & -1.59 & 705 & 725 & E2W2 & 155.62 & -111.65 & $0.153_{ \pm 0.024 \pm 0.002}$ \\
\hline & 2013.10 .26 & 56591.919 & -1.59 & 725 & 740 & E2W2 & 155.62 & -111.65 & $0.118_{ \pm 0.021 \pm 0.001}$ \\
\hline & 2014.10 .16 & 56947.000 & -0.25 & 695 & 715 & W1W2 & 104.65 & -86.71 & $0.504_{ \pm 0.061 \pm 0.006}$ \\
\hline & 2014.10 .16 & 56947.019 & 0.19 & 695 & 715 & W1W2 & 106.99 & -86.35 & $0.419_{ \pm 0.049 \pm 0.005}$ \\
\hline & 2014.10.18 & 56948.965 & -0.96 & 695 & 715 & W1W2 & 98.08 & -87.20 & $0.482_{ \pm 0.030 \pm 0.004}$ \\
\hline \multirow[t]{2}{*}{ HD 5268} & 2013.07 .28 & 56501.961 & -1.05 & 690 & 710 & W2S2 & 124.28 & 163.39 & $0.388_{ \pm 0.077 \pm 0.009}$ \\
\hline & 2013.07.28 & 56501.961 & -1.05 & 710 & 730 & W2S2 & 124.28 & 163.39 & $0.336_{ \pm 0.072 \pm 0.008}$ \\
\hline
\end{tabular}

Notes. The columns list the date, the reduced Julian date (RJD = JD - 2400 000), the hour angle (HA), the minimum and maximum wavelengths over which the squared visibility is calculated, the projected baseline length Bp, and its orientation PA. The last column provides the calibrated squared visibility $V^{2}$ together with the statistic error on $V^{2}$ and the systematic error on $V^{2}$ (see text for details). Only the visibilities with a signalto-noise ratio on the fringe peak higher than 1.7 were considered. The data are available on the Jean-Marie Mariotti Center OiDB service (available at http://oidb.jmmc.fr). 